\title{
A Note on Mod and Generalised Mod Classes
}

\author{
Meena Mahajan* $\quad$ N. V. Vinodchandran $^{\dagger}$
}

Keywords. Computational Complexity: Mod classes, relativised separations, truth-table reductions.

\section{Introduction}

We characterise $M o d$ classes in terms of \#P functions, where the membership is determined by co-primality or gcd testing of the function value (Theorem 3.1), instead of residue $(\bmod k)$ testing. Imposing a restriction on the range of the functions gives a characterisation of the intersection of Mod classes (Theorem 3.2). These intersection classes, which we denote by $\operatorname{Mod} \cap_{k} P$, are interesting because they share most of the "nice" properties (closure under complementation, normal forms, lowness for itself etc) of $\operatorname{Mod}_{p} P$ for prime $p$. We show that the class $\operatorname{Mod} \cap_{k} P$ is low for $\operatorname{Mod}_{k} P$, and also for $\operatorname{Mod} \cap_{k} P$ itself (Theorem 3.3).

We also strengthen some of the separation results known for Mod classes. A diagonalisation argument due to Beigel shows that when $k$ is a prime not dividing $j, \operatorname{Mod}_{j} P$ can be separated from $\operatorname{Mod}_{k} P$ in some relativised world. We observe that this argument even separates $\operatorname{Mod} \cap_{j} P$ from $\operatorname{Mod}_{k} P$ under the same conditions (Theorem 4.1). Further, if $k$ is not known to be prime, the same argument still diagonalises, but out of a smaller class; it separates $\operatorname{Mod} \cap_{j} P$ from $\operatorname{Mod} \cap_{k} P$ (Theorem 4.2).

The class $M o d P$ was defined in [6] as a generalisation of the Mod classes. We define a simple generalisation, $\operatorname{ModK} P$, and show that it coincides with the disjunctive truth table closure of $M o d P, P_{d t t}^{M o d P}$ (Theorem 5.2). We give neat characterisations of $P_{d t t}^{M o d P}$ and $P_{c t t}^{M o d P}$ (Theorem 5.3), and also a new characterisation of ModP (Theorem 5.4).

The results of section 5 thus give us an overall picture of the relations between the generalised Mod classes as shown in Figure 1. Arrows denote containment, and connections tagged coindicate that the corresponding classes are the Co-classes of each other.

\footnotetext{
*The Institute of Mathematical Sciences, Madras 600 113, India. email: meena@imsc.ernet.in

${ }^{\dagger}$ The Institute of Mathematical Sciences, Madras 600 113, India. email: vinod@imsc.ernet.in. Work done at the Department of Computer Science and Engineering, Indian Institute of Technology, Madras 600 036, India.
} 


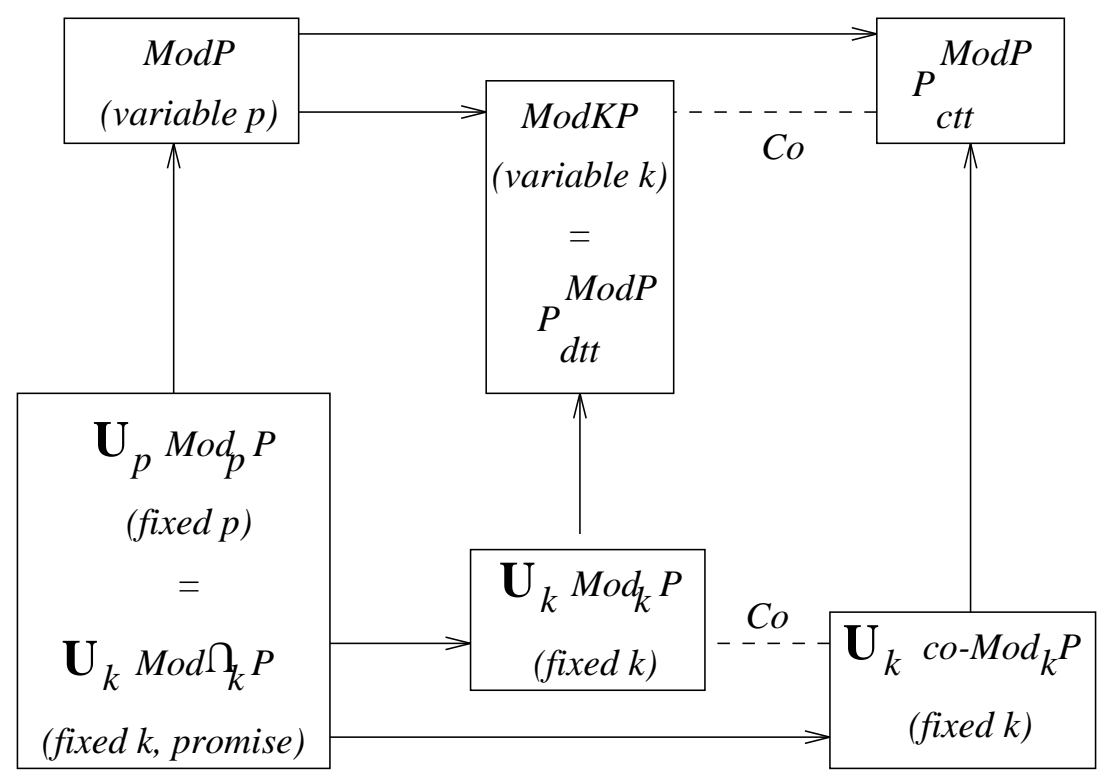

Figure 1. Relations among gemeralised Mod classes 


\section{Preliminaries}

We follow the standard definitions and notations in computational complexity theory (see, e.g., [1] or [5]). A function $f$ is in \#P if there exists a nondeterministic polynomial time Turing Machine $M$ such that $\forall x, f(x)$ is the number of accepting paths of $M$ on input $x$. \#P is closed under several arithmetic operations, including addition, multiplication, binomial coefficients, etc. [3]. A language $L$ is in $\operatorname{Mod}_{k} P[3,4]$ if there is a \#P function $f$ such that $\forall x \in \Sigma^{*}, x \in$ $L \Leftrightarrow f(x) \not \equiv 0 \quad(\bmod k)$. We use the notation $\vee_{i} \operatorname{Mod}_{p_{i}} P$ and $\wedge_{i} \operatorname{Mod}_{p_{i}} P$ to denote the classes $\left\{\cup_{i} L_{i} \mid L_{i} \in \operatorname{Mod}_{p_{i}} P\right\}$ and $\left\{\cap_{i} L_{i} \mid L_{i} \in \operatorname{Mod}_{p_{i}} P\right\}$ respectively. If $k$ has prime factorisation $k=$ $\Pi_{i} p_{i}^{\alpha_{i}}$ where the $p_{i}$ are distinct primes, then we denote $\Pi_{i} p_{i}$ by $\pi(k)$. If $\pi(k)=k$ then $k$ is said to be squarefree. $\phi(k)$ denotes the Eulerian function, the number of integers less than and co-prime to $k$.

The following results about Mod classes will be used in this note.

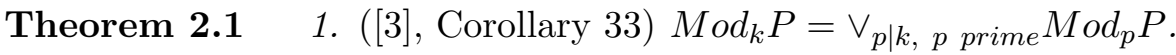

2. ([3], Theorem 23) Let $p$ be prime. A language $L$ is in $\operatorname{Mod}_{p} P$ if and only if it has a 0-1 normal form \#P function; that is, there exists a \#P function satisfying, for all $x$,

$$
\begin{aligned}
& x \in L \quad \Rightarrow \quad f(x) \equiv 1 \quad(\bmod p) \\
& x \notin L \quad \Rightarrow \quad f(x) \equiv 0 \quad(\bmod p)
\end{aligned}
$$

3. ([3], Theorem 27) If $p$ is prime, then $\operatorname{Mod}_{p} P^{\operatorname{Mod}_{p} P}=\operatorname{Mod}_{p} P$.

4. ([2], Theorem 10) Let $j>1$, and let $k$ be a prime number that is not a divisor of $j$. There exists an oracle $A$ such that $\operatorname{Mod}_{j} P^{A} \nsubseteq \operatorname{Mod}_{k} P^{A}$.

The class $M o d P$ has been introduced by Köbler et al in [6]. ModP is the generalised version of $\operatorname{Mod}_{p} P$, where $p$ is a prime.

Definition $2.2([6])$ A language $L$ is in ModP iff there exists a \#P function $f$ and a function $g \in F P$ such that for all strings $x, g(x)=0^{p}$ for some prime $p$, and $x \in L \Leftrightarrow f(x) \not \equiv 0$ $(\bmod p)$.

It is shown in $[6]$ that the \#P function $f$ can be brought into $0-1$ normal form (it always evaluates to either 1 or $0 \quad(\bmod |g(x)|))$. It has also been shown that the class does not change if, in the definition, $g$ is allowed to return powers of prime numbers. 


\section{New Characterisations}

In this section, we show some new characterisations for Mod classes. These characterisations use co-primality and gcd testing on the values of \#P functions, rather than residue testing.

Theorem 3.1 $L \in \operatorname{Mod}_{k} P$ if and only if there exists a \#P function $f$ such that $x \in L \Leftrightarrow$ $\operatorname{gcd}(f(x), k) \neq 1$.

Proof: $(\Rightarrow)$. Let $L \in \operatorname{Mod}_{k} P$. Let $k=\Pi_{i} p_{i}^{\alpha_{i}}$, where $p_{i}$ are the prime factors of $k$. Then

$$
L \in \vee_{p_{i} \mid k, p_{i} \text { prime } M_{o d} P} P \text { Theorem 2.1, } 1 .
$$

$\Rightarrow \bar{L} \in \wedge_{p_{i} \mid k, p_{i} \text { prime }} \mathrm{Co-} \operatorname{Mod}_{p_{i}} P$

$\Rightarrow \bar{L} \in \wedge_{p_{i} \mid k, p_{i}}$ prime $^{M_{\text {od }}}{ }_{p_{i}} P$

So let $\bar{L}=\cap L_{i}$ where each $L_{i} \in \operatorname{Mod}_{p_{i}} P$ via \#P function $f_{i}$ in $0-1$ normal form (from Theorem 2.1,2). Then it is easy to verify that the function $f=\Sigma_{i} \frac{k}{p_{i}^{\alpha_{i}}} f_{i}$ satisfies the given conditions.

$(\Leftarrow)$. If $f$ is a \#P function satisfing the given conditions then $L \in \operatorname{Mod}_{k} P$ via the $\# P$ function $h=f^{\phi(k)}+(k-1)$.

In other words, a language $L \in \operatorname{Mod}_{k} P$ can be characterised using a \#P function $f$ such that if $x \in L, f$ maps $x$ to a non-invertible element of the $\operatorname{ring} \mathbf{Z} / k \mathbf{Z}$, and if $x \notin L, f$ maps $x$ to an invertible element.

A promise version of the above class of functions, where

$$
\operatorname{gcd}(f(x), k) \neq 1 \Rightarrow \operatorname{gcd}(f(x), k)=k
$$

characterises the intersection of the $\operatorname{Mod}_{p_{i}} P$ classes, where $p_{i}$ is a prime factor of $k$. For brevity we henceforth denote this class by

$$
\operatorname{Mod} \cap_{k} P \triangleq \cap_{p_{i} \mid k, p_{i} \text { prime }} \operatorname{Mod}_{p_{i}} P
$$

Thus if $k$ is squarefree, then $\operatorname{Mod} \cap_{j} P=\operatorname{Mod} \cap_{k} P$ for all $j$ such that $\pi(j)=k$.

Theorem 3.2 $L \in M o d \cap_{k} P$ iff there exists a \#P function $f$ such that

$$
\begin{aligned}
& x \in L \quad \Rightarrow \quad \operatorname{gcd}(f(x), k)=1 \\
& x \notin L \Rightarrow \operatorname{gcd}(f(x), k)=k
\end{aligned}
$$

Proof: $(\Rightarrow)$. Let $L \in M o d \cap_{k} P$ where $k=\Pi_{i} p_{i}^{\alpha_{i}}$. For all $i$, let $L \in \operatorname{Mod}_{p_{i}} P$ via $\# P$ functions $h_{i}$ in 0-1 normal form. Then $L \in \operatorname{Mod}_{p_{i}^{\alpha_{i}}} P$ via $\# P$ function $f_{i}=h_{i}^{\alpha_{i} \phi\left(p_{i}^{\alpha_{i}}\right)}$ which is also in 0-1 normal form. Now it is easy to verify that the function $f=\Sigma_{i} \frac{k}{p_{i}^{\alpha_{i}}} f_{i}$ satisfies the given conditions. 
$(\Leftarrow)$. It is obvious that if there exists a function satisfying the conditions, then $L \in \operatorname{Mod}_{p_{i}} P$ for each $i$ via the same function. Hence $L \in \operatorname{Mod} \cap_{k} P$.

It follows that for every $k$, languages in $\operatorname{Mod} \cap_{k} P$ have a 0-1 normal form \#P function with respect to $k$. Note that in the above theorem, the conditions of Theorem 3.1 have been restricted to the promise version and inverted. This does not matter because $\operatorname{Mod} \cap_{k} P$ is closed under complementation.

The class $\operatorname{Mod} \cap_{k} P$ is of some interest because it is low for $\operatorname{Mod}_{k} P$, as we show below. In fact, it is also low for itself, whereas an analogous result for $\operatorname{Mod}_{k} P$ classes is known to hold only when $k$ is prime. Also, we do not know of any class which contains $\operatorname{Mod} \cap_{k} P$ and is low for $\operatorname{Mod}_{k} P ; \operatorname{Mod} \cap_{k} P$ is the largest known class with this property.

Theorem 3.3 For any $k \geq 2$,

(1) $\operatorname{Mod}_{k} P^{M o d \cap_{k} P}=\operatorname{Mod}_{k} P$

(2) $\operatorname{Mod} \cap_{k} P^{M o d \cap_{k} P}=\operatorname{Mod} \cap_{k} P$

Proof: We prove (1); (2) follows identically. Let $A \in \operatorname{Mod}_{k} P^{M o d \cap_{k} P}$ via an oracle $L \in$ $\operatorname{Mod} \cap_{k} P$. Then

$$
\begin{array}{rlr}
A & \in \vee_{p_{i} \mid k, p_{i} \operatorname{prime} \operatorname{Mod}_{p_{i}} P^{L}} & \text { relativised version of Theorem 2.1, } 1 . \\
& \subseteq \vee_{p_{i} \mid k, p_{i} \text { prime } \operatorname{Mod}_{p_{i}} P^{M o d} p_{p_{i}} P} & \text { by definition of } \operatorname{Mod} \cap_{k} P \\
& =\vee_{p_{i} \mid k, p_{i} \text { prime } \operatorname{Mod}_{p_{i}} P} & \text { Theorem 2.1,3. } \\
& =\operatorname{Mod}_{k} P & \text { Theorem 2.1, } 1 .
\end{array}
$$

\section{Separation Results}

In [2], the construction of an oracle relative to which $\operatorname{Mod}_{j} P$ is not contained in $\operatorname{Mod}_{k} P$ is outlined (Theorem 2.1,4.). This result applies when $k$ is prime and $j$ and $k$ are relatively prime. It is open whether the second condition alone is sufficient to exhibit such a separation. If we consider separations of the Mod $\cap$ classes instead of Mod classes, then we show (Theorem 4.2) that this condition suffices.

A careful examination of the oracle construction in [2] shows that only the subset $\operatorname{Mod} \cap_{j} P$ of $\operatorname{Mod}_{j} P$ is used in proving $\operatorname{Mod}_{j} P^{A} \nsubseteq \operatorname{Mod}_{k} P^{A}$. The construction diagonalises out of the class $\operatorname{Mod}_{k} P$, in the process creating a language which satisfies the promise of Theorem 3.2. Thus the construction actually proves the following result: 
Theorem 4.1 Let $j>1$, and let $k$ be a prime that is not a divisor of $j$. Then there exists an oracle $A$ such that

$$
\operatorname{Mod} \cap_{j} P^{A} \nsubseteq \operatorname{Mod}_{k} P^{A}
$$

If $k$ is allowed to be composite, as long as it has at least one prime factor not dividing $j$, the diagonalisation argument can still be used. However, it now diagonalises out of a much smaller (presumably) class, namely the class $\operatorname{Mod} \cap_{k} P$.

Theorem 4.2 Let $j$ and $k$ be two integers. If $k$ has a prime factor not dividing $j$, then there exists an oracle $B$ such that $\operatorname{Mod} \cap_{j} P^{B} \nsubseteq \operatorname{Mod} \cap_{k} P^{B}$.

Proof: Since $k$ has a prime factor $p$ that does not divide $j$, it follows from the above theorem that there exists an oracle $B$ such that $\operatorname{Mod} \cap_{j} P^{B} \nsubseteq \operatorname{Mod}_{p} P^{B}$. But $\operatorname{Mod}_{k} P^{B} \subseteq \operatorname{Mod}_{p} P^{B}$, since $p \mid k$. Therefore $\operatorname{Mod} \cap_{j} P^{B} \nsubseteq \operatorname{Mod} \cap_{k} P^{B}$.

In particular, if $\operatorname{gcd}(j, k)=1$, then the corresponding $\operatorname{Mod} \cap P$ classes can be separated; there exists an oracle $B$ such that $\operatorname{Mod} \cap_{j} P^{B} \nsubseteq \operatorname{Mod} \cap_{k} P^{B}$.

For any two primes $p, q$, the classes $\operatorname{Mod}_{p} P$ and $\operatorname{Mod}_{q} P$ can be separated (from Theorm 2.1, 4.) in some relativised world. Consequently, we have a proper separation between the $\operatorname{Mod} \cap_{k} P$ and $\operatorname{Mod}_{k} P$ classes in some relativised world, as the following corollary states .

Corollary 4.3 If $k$ is not prime or a power of a prime, then there is an oracle $C$ such that $\operatorname{Mod} \cap_{k} P^{C} \subset \operatorname{Mod}_{k} P^{C}$.

\section{$5 \quad$ Generalised Mod classes}

In this section we generalise the class $\operatorname{Mod}_{k} P$ to $\operatorname{ModKP}$ and show that this class is precisely the disjunctive truth table closure of the class ModP.

Definition 5.1 A language $L$ is in ModKP iff there exists a \#P function $f$ and a function $g \in F P$ such that for all strings $x, g(x)$ outputs a positive integer $k$ as a list $\left\langle 0^{p_{1}^{\alpha_{1}}}, 0^{p_{2}^{\alpha_{2}}}, \cdots, 0^{p_{n}^{\alpha_{n}}}\right\rangle$ where $k=\Pi_{i} p_{i}^{\alpha_{i}}$, and $x \in L \Leftrightarrow f(x) \not \equiv 0 \quad(\bmod k)$.

( $k$ can also be represented as a list $\left\langle\left\langle 0^{p_{1}}, 0^{\alpha_{1}}\right\rangle,\left\langle 0^{p_{2}}, 0^{\alpha_{2}}\right\rangle, \cdots,\left\langle 0^{p_{n}}, 0^{\alpha_{n}}\right\rangle\right\rangle$. Even though $0^{p_{i}^{\alpha_{i}}}$ requires $p_{i}^{\alpha_{i}}$ to be polynomially bounded (implying small exponents), the same number $p_{i}^{\alpha_{i}}$ with polynomial-valued $\alpha_{i}$ can be expressed simply by repeating $0^{p_{i}} \alpha_{i}$ times in the list.) 
Theorem 5.2 $P_{d t t}^{M o d P}=M o d K P$

Proof: (a) $M o d K P \subseteq P_{d t t}^{M o d P}$.

Let $L \in M o d K P$ via $f \in \# P$ and $g \in F P$. Define $B=\left\{\left\langle x, 0^{p^{e}}\right\rangle \mid f(x) \not \equiv 0 \quad\left(\bmod p^{e}\right)\right\}$. Then $B \in M o d P$ via $\# P$ function $f$ and $F P$ function $g_{B}$, where $g_{B}$, on input $\left\langle x, 0^{p^{e}}\right\rangle$, outputs $0^{p^{e}}$. (Although $g$ does not return a prime, it always returns a power of a prime. So the language is still in $\operatorname{ModP}$, as described in [6].)

Let $g(x)=\left\langle 0^{p_{1}^{\alpha_{1}}}, 0^{p_{2}^{\alpha_{2}}}, \cdots, 0^{p_{n}^{\alpha_{n}}}\right\rangle$, representing $k=\Pi_{i} p_{i}^{\alpha_{i}}$. Now $L$ disjunctively reduces to $B$ via an FP function $h$, where $h$, on input $x$, produces the list $\left\langle\left\langle x, 0^{p_{1}^{\alpha_{1}}}\right\rangle,\left\langle x, 0^{p_{2}^{\alpha_{2}}}\right\rangle, \cdots,\left\langle x, 0^{p_{n}^{\alpha_{n}}}\right\rangle\right\rangle$. (b) $P_{d t t}^{M o d P} \subseteq M o d K P$.

Let $L$ be disjunctively reducible to a set $B \in \operatorname{ModP}$ via $h$. Then for all strings $x, h(x)$ produces a list $\left\langle y_{1}, y_{2}, \cdots, y_{m}\right\rangle$ such that $x \in L \Leftrightarrow \exists i, 1 \leq i \leq m: y_{i} \in B$.

Let $B \in M o d P$ via a $0-1$ normal form \#P function $f$ and an $F P$ function $g$. For any string $x$, let $P(x)=\left\{\left|g\left(y_{1}\right)\right|,\left|g\left(y_{2}\right)\right| \cdots\left|g\left(y_{m}\right)\right|\right\}$ be the set of primes computed by $g$. (Note that two strings may give the same prime on same input $x$.) Let $I_{p}(x)=\left\{y_{i} \mid g\left(y_{i}\right)=0^{p}\right\}$. Define functions $\tilde{f}$ and $\tilde{g}$ as follows:

$$
\begin{gathered}
\tilde{f}=\sum_{p \in P(x)}\left\{\left(\prod_{q \in P(x)-p} q\right)\left(\left(\prod_{y \in I_{p}(x)}(f(y)+p-1)^{p-1}\right)(p-1)+1\right)\right\} \\
\tilde{g}(x)=\left\langle 0^{p_{1}}, 0^{p_{2}}, \cdots, 0^{p_{n}}\right\rangle \text { each } p_{i} \in P(x)
\end{gathered}
$$

Since the value of each prime is polynomial in the length of $x$, it follows from the closure properties of $\# P$ functions that $\tilde{f} \in \# P$. Also it is easy to verify that $\tilde{g} \in F P$. We show that the language $L \in M o d K P$ via $\tilde{f} \in \# P$ and $\tilde{g} \in F P$.

Let the value that $\tilde{g}$ computes on input $x$ be $k$.

$$
\begin{aligned}
x \in L & \Rightarrow f\left(y_{i}\right) \equiv 1 \quad\left(\bmod \left|g\left(y_{i}\right)\right|\right) \text { for some } i \leq m \\
& \Rightarrow f\left(y_{i}\right) \equiv 1 \quad(\bmod p) \text { for some } p \in P(x), p=\left|g\left(y_{i}\right)\right| \\
& \Rightarrow \quad\left(\prod_{y \in I_{p}(x)}(f(y)+p-1)^{p-1}\right)(p-1)+1 \equiv 1 \quad(\bmod p) \\
& \Rightarrow \tilde{f}(x) \not \equiv 0 \quad(\bmod p) \\
& \Rightarrow \tilde{f}(x) \not \equiv 0 \quad(\bmod k) \\
x \notin L & \Rightarrow f\left(y_{i}\right) \equiv 0 \quad\left(\bmod \left|g\left(y_{i}\right)\right|\right) \text { for all } i \leq m \\
& \Rightarrow f\left(y_{i}\right) \equiv 0 \quad(\bmod p), p=\left|g\left(y_{i}\right)\right| \forall i \\
& \Rightarrow\left(\prod_{y \in I_{p}(x)}(f(y)+p-1)^{p-1}\right)(p-1)+1 \equiv 0 \quad(\bmod p) \forall p \in P(x) \\
& \Rightarrow \tilde{f}(x) \equiv 0 \quad(\bmod k)
\end{aligned}
$$


Like $\operatorname{Mod}_{k} P, \operatorname{ModKP}$ can also be characterised in terms of gcd testing.

Theorem 5.3 A language $L \in M o d K P$ if and only if there exists a\#P function $f$ and a function $g \in F P$ such that for all strings $x, g(x)$ outputs a positive integer $k$ as a list $\left\langle 0^{p_{1}^{\alpha_{1}}}, 0^{p_{2}^{\alpha_{2}}}, \cdots, 0^{p_{n}^{\alpha_{n}}}\right\rangle$ where $k=\Pi_{i} p_{i}^{\alpha_{i}}$, and

$$
x \in L \Leftrightarrow \operatorname{gcd}(f(x), k) \neq 1
$$

Proof: (a) Let $L$ be in $M o d K P$ via functions $h \in \# P, g \in F P$. Then $f$ as defined below satisfies the required condition. Let $k=\Pi_{i} p_{i}^{\alpha_{i}}$ be computed by $g$ on input $x$. Then

$$
f(x)=\Sigma_{i} \frac{k}{p_{i}^{\alpha_{i}}}\left[(h(x))^{p_{i}-1}\left(p_{i}-1\right)+1\right] .
$$

(b) Checking if $\operatorname{gcd}(f(x), k)=1$ is conjunctive truth-table reducible to $\operatorname{ModP}$ queries (using a construction similar to that in the proof of Theorem 5.2 (a)). Since ModP is closed under complementation [6], $M o d K P=P_{d t t}^{M o d P}=\operatorname{co}-P_{c t t}^{M o d P}$.

The preceding theorem also characterises $P_{c t t}^{M o d P}$ as the class of languages $L$ such that $x \in$ $L \Leftrightarrow \operatorname{gcd}(f(x), k)=1$, where $f$ and $g$ are as defined in the theorem.

For $M o d P$, the \#P function can be brought into 0-1 normal form. If the $F P$ function is also allowed to return non-primes in suitably encoded form, we get the presumably larger class $M o d K P=P_{d t t}^{M o d P}$. However, if the \#P function is constrained to be in 0-1 normal form with respect to these composite numbers as well, we get back the original class $\operatorname{ModP}$, as shown below.

Theorem 5.4 A language $L \in M o d P$ if and only if there exists a function $f \in \# P$ and $a$ function $g \in F P$ such that for all strings $x, g(x)=0^{k}$ for some positive integer $k \geq 2$ and

$$
\begin{aligned}
& x \in L \quad \Rightarrow \quad f(x) \equiv 1 \quad(\bmod k) \\
& x \notin L \quad \Rightarrow \quad f(x) \equiv 0 \quad(\bmod k)
\end{aligned}
$$

(Larger values of $k$ can be represented using the list representation, as in the definition of ModKP. However, it is easy to see that in this case, this makes no difference to the class.) Proof: $(\Rightarrow)$ This follows from the 0-1 normal form of ModP.

$(\Leftarrow)$ Suppose there exist functions $f \in \# P$ and $g \in F P$. Consider the function $g^{\prime}$ which returns any prime factor of the number computed by $g$ on $x$. Since $g$ returns $g(x)$ in unary (or in factorised) notation, clearly $g^{\prime} \in F P$. Now $L \in M o d P$ via $f$ and $g^{\prime}$. 


\section{Acknowledgements}

We wish to thank Johannes Köbler for pointing out an error in an earlier version of this note. We also wish to thank V Arvind and V Vinay for many comments which helped improve the presentation and readability of this note.

\section{References}

[1] J. L. Balcázar, J. Díaz, and J. Gabarró. Structural Complexity - I. Springer Verlag, Berlin Heidelberg, 1988.

[2] R. Beigel. Relativized counting classes: relations among thresholds, parity, and mods. Journal of Computer and System Sciences, 42:76-96, 1991.

[3] R. Beigel, J. Gill, and U. Hertrampf. Counting classes: Thresholds, parity, mods, and fewness. In Proceedings of the Seventh Annual Symposium on Theoretical Aspects of Computer Science, pages 49-57. Springer-Verlag, 1990. Lecture Notes in Computer Science \# 415.

[4] U. Hertrampf. Relations among Mod-classes. Theoretical Computer Science, 74:325-328, 1990.

[5] J. Hopcroft and J. Ullman. Introduction to Automata Theory, Languages, and Computation. Addison-Wesley, 1979.

[6] J. Köbler and S. Toda. On the power of generalized MOD-classes. In Proceedings of the Eighth Annual Conference on Structure in Complexity theory, 1993. 